\title{
HMGB1 enhances chemotherapy resistance in multiple myeloma cells by activating the nuclear factor- $\kappa B$ pathway
}

\author{
JING NING, RUI YANG, HAINAN WANG and LIJUAN CUI \\ Department of Hematology, The General Hospital of Ningxia Medical University, \\ Yinchuan, Ningxia Hui Autonomous Region 750004, P.R. China
}

Received October 23, 2020; Accepted March 31, 2021

DOI: $10.3892 /$ etm.2021.10137

\begin{abstract}
Chemotherapy resistance is a main obstacle in the clinical chemotherapeutic treatment of multiple myeloma (MM). High-mobility group box 1 (HMGB1) has been revealed to be associated with the sensitivity of MM cells to chemotherapy, but how HMGB1 regulates chemotherapy resistance in $\mathrm{MM}$ has yet to be fully elucidated. In the present study, the exact molecular mechanism underlying HMGB1-mediated drug resistance in MM was explored using three chemotherapy-resistant MM cells (RPMI8226/ADR, RPMI8226/BOR and RPMI8226/DEX) that were successfully established. Reverse transcription-quantitative polymerase chain reaction revealed that the three chemotherapy-resistant MM cells exhibited a higher release of HMGB1 compared with the parental RPMI8226 cells. Interference with endogenous HMGB1 increased the sensitivity of drug-resistant MM cells to chemotherapy, which was supported by the low $\mathrm{IC}_{50}$ value and the enlargement of cell apoptosis. Furthermore, short hairpin (sh)RNA-transfected MM cells showed an obvious elevation in phosphorylated (p)-IKK $\alpha / \beta, \mathrm{p}-\mathrm{I} \kappa \mathrm{B} \alpha$ and p-p65 in whole cell lysate and/or nucleus, and treatment of nuclear factor $(\mathrm{NF})-\kappa \mathrm{B}$ activator reversed the effect of shHMGB1-mediated cell viability and apoptosis in MM cells. In conclusion, HMGB1 regulates drug resistance in $\mathrm{MM}$ cells by regulating $\mathrm{NF}-\kappa \mathrm{B}$ signaling pathway, suggesting that HMGB1 has the potential to serve as a target for MM treatment.
\end{abstract}

\section{Introduction}

Multiple myeloma (MM) is a serious hematological malignancy characterized by mass aggregation of malignant plasma cells in the bone marrow and the existence of monoclonal

Correspondence to: Dr Lijuan Cui, Department of Hematology, The General Hospital of Ningxia Medical University, 804 Shengli Street, Xingqing, Yinchuan, Ningxia Hui Autonomous Region 750004, P.R. China

E-mail: cuilijuan7511@163.com

Key words: HMGB1, multidrug resistance, multiple myeloma, nuclear factor- $\kappa \mathrm{B}$ pathway protein (M protein) in blood, urine, or both (1). To improve the life quality and prolong the survival time of patients with MM, various available therapeutic options including chemotherapy, autologous/allogeneic stem cell transplantation and targeted drug therapy have been widely applied in the clinic to treat this disease (2). In the last few decades, great advancement in cellular and molecular mechanisms of MM has been made, leading to the development of a wide range of new drugs, such as adriamycin (ADR), bortezomib (BOR) and dexamethasone (DEX) (3). Although these drugs had significantly improved the quality of life of patients to some extent, the clinical outcome remains unsatisfactory because of acquired drug resistance, which has become the main obstacle of the failure of chemotherapy in the clinical treatment of MM. Therefore, the further exploration of the potential molecular mechanism underlying the acquired chemotherapy resistance in MM is extremely urgent for seeking effective treatment strategies for MM.

High-mobility group box 1 (HMGB1), an important nonhistone protein in the nucleus, functions as a key moderator in DNA arrangement, replication, damage repair and transcription by stabilizing nucleosome function (4). An obvious upregulation of HMGB1 has been identified in several types of tumor tissues compared with that of the corresponding normal tissues, which is linked with the tumor development and progression, including inflammation and angiogenesis, invasion, progression and metastasis $(5,6)$. Moreover, HMGB1 can be released from diverse tumor cells in response to chemotherapy or radiotherapy, and thus participates in the regulation of the chemoresistance and sensitivity of cancer cells $(7,8)$. The study of Liu et al (9) found that the release of HMGB1 from leukemia cell lines increased under the stimulation of chemotherapy, and treatment with HMGB1-neutralizing antibodies rendered these cells more sensitive to chemotherapy. One in vivo experiment revealed that HMGB1 can promote tumor growth by enhancing the drug resistance of lung cancer cells (7). In human lung adenocarcinoma, HMGB1-regulated autophagy is also considered as a critical contributor to docetaxel resistance (10). In MM cells, HMGB1 was upregulated and its expression was negatively associated with the survival of patients with MM; meanwhile, bortezomibresistant MM cells also exhibited a higher HMGB1 expression and HMGB1 knockdown can enhance the sensitivity of MM cells to chemotherapy in vivo $(11,12)$. However, the association 
between HMGB1 and MM chemoresistance, and the potential molecular mechanism has not been clarified thoroughly.

Nuclear factor- $\kappa \mathrm{B}(\mathrm{NF}-\kappa \mathrm{B})$, a tightly regulated transcription factor, functions as a moderator in almost all types of cancer development (13). NF- $\mathrm{NB}$ signaling was found to be constitutively activated in some tumor cells, including leukemia (14), lung (15), lymphoma (16) and breast (17) cancer cell lines. Besides, the increased NF- $\kappa \mathrm{B}$ levels indicate a poor prognosis in glioblastoma (18) and ovarian cancer (19), and the blockage of $\mathrm{NF}-\kappa \mathrm{B}$ signaling has been demonstrated to exhibit an anti-tumor response (19). Except for the role in cancer development, activated $\mathrm{NF}-\kappa \mathrm{B}$ has also been recognized as a key regulator in chemoresistance. Increasing studies have supported that the chemotherapy-resistant tumor cells showed significantly higher expression of $\mathrm{NF}-\kappa \mathrm{B}$ compared with the matched parental cells. In carcinoma cell lines, $\mathrm{NF}-\kappa \mathrm{B}$ activity was found to be negatively associated with cellular sensitivity to chemotherapy (20); in several lung cell lines, long term treatment with increasing doses of cisplatin would render them becoming resistant to cisplatin (21). The aforementioned data revealed that $\mathrm{NF}-\kappa \mathrm{B}$ signaling acts as a potential mediator in the development of acquired cisplatin resistance.

A gene expression profiling based on 320 diagnosed patients with MM identified the engagement and significance of NF- $\kappa \mathrm{B}$ signaling in MM cells (22), and the gene mutations involving this pathway occurred on at least $40 \%$ of MM cell lines (MMCLs) and 17\% of MM tumors $(23,24)$. NF- $\kappa \mathrm{B}$ signaling has been identified to function as a facilitator in proliferation and drug-resistance of myeloma cells, which played an important role in the interactions of the myeloma cells with the bone marrow microenvironment (25). However, the exact molecular mechanism of $\mathrm{NF}-\kappa \mathrm{B}$ in the development by which the MM cell develops acquired resistance remains to be explored.

A previous study identified the important role of HMGB1 in the occurrence and development of diverse tumors through the NF- $\kappa$ B signaling pathway (26). Thus, it is postulated that HMGB1 is most likely to be involved in the regulation of MM chemoresistance via NF- $\mathrm{BB}$ signaling. To verify this, the present study established adriamycin-, bortezomib- and dexamethasone-resistant MM cells, and then a series of experiments was performed to confirm the hypothesis. The present study may help to better understand the molecular mechanisms of MM chemoresistance, and further develop novel therapeutic strategies in the treatment of MM.

\section{Material and methods}

Cell culture. The MMCL RPMI8266 was purchased from the American Type Culture Collection. All cells were maintained in RPMI1640 containing 10\% fetal bovine serum (Gibco; Thermo Fisher Scientific, Inc.) at $37^{\circ} \mathrm{C}$ in a humidified atmosphere with $5 \% \mathrm{CO}_{2}$.

Cell transfection. Short hairpin RNA (shRNA) against human HMGB1 (Sigma-Aldrich; Merck KGaA) and control shRNA (Sigma-Aldrich; Merck KGaA) were transfected into cells using the Lipofectamine ${ }^{\circledR} 2000$ reagent (Thermo Fisher Scientific, Inc.) according to the manufacturer's instructions. Briefly, a mixture of $1 \mathrm{ml}$ Lipofectamine 2000 and
50 ml Opti-MEM I (Gibco; Thermo Fisher Scientific, Inc.) was added to the serum-free medium when the cell density reached $\sim 50 \%$. After $10 \mathrm{~min}$, serum-free medium was mixed with $1 \mathrm{mg}$ FAM-shRNA and $50 \mathrm{ml}$ Opti-MEM I. After incubating at room temperature for 5 min, diluted FAM-shRNA and Lipofectamine 2000 were mixed and incubated for $20 \mathrm{~min}$. The successfully transfected cells were screened with $0.3 \mu \mathrm{g} / \mathrm{ml}$ puromycin. The interference sequence against HMGB1 was 5'-GGACAAGGCCCGTTATGAA-3', and the control sequence was 5'-TTCTCCGAACGTGTACGT-3'. At $48 \mathrm{~h}$ post transfection, transfected cells were collected for next analysis.

Establishment of drug-resistance cell lines. The parental RPMI8266 cells $\left(5 \times 10^{5}\right)$ were seeded into 6-well plates containing $10 \mathrm{nM}$ bortezomib (Sigma-Aldrich; Merck KGaA) when they reached the exponential growth phase, and were incubated in $37^{\circ} \mathrm{C}$ with $5 \% \mathrm{CO}_{2}$ condition. The cell culture medium was replaced every 2-3 days until the cells return to the normal growth state. After 1-2 weeks, bortezomib (10 nM) was added to the culture medium again; when this process has been repeated for three times, the final concentration of bortezomib was increased to $20 \mathrm{nM}$. The aforementioned steps were repeated until the bortezomib concentration was increased to $200 \mathrm{nM}$. After 8-10 months, the bortezomibresistant RPMI8266 cells were obtained. A similar approach was also used to establish adriamycin- and dexamethasoneresistant cell lines. Finally, ADR-resistant RPMI8226 cells (160 nM of ADR), BOR-resistant RPMI8226 cells (200 nM of BOR), and DEX-resistant RPMI8226 cells (180 nM of DEX) were successfully established.

Cell cycle analysis. Cells $\left(2 \times 10^{5}\right.$ cells/well) were cultured in sixwell plates for $48 \mathrm{~h}$. Then, the cells were washed with PBS and permeabilized with precooled $75 \%$ ethanol at $4^{\circ} \mathrm{C}$ overnight. After washing, the cells were incubated with $500 \mu \mathrm{l}$ propidium iodide (PI; $5 \mathrm{mg} / \mathrm{ml}$; Sigma-Aldrich; Merck KGaA) for $30 \mathrm{~min}$ at room temperature, according to the manufacturer's instructions. The alterations in cell cycle were determined by using the program M software (System II Software; version 3.0) on an Epics flow cytometer (Coulter Immunology).

Cell apoptosis analysis. RPMI8226, RPMI8226/ADR, RPMI8226/BOR and RPMI8226/DEX cells were respectively treated with a specific concentration of ADR (160 nM), BOR (200 nM) and DEX (180 nM) for $48 \mathrm{~h}$. Subsequently, the cells $\left(1 \times 10^{5}\right)$ were incubated with Annexin V-FITC and propidium iodide (PI; Beyotime Institute of Biotechnology). Finally, the degree of cell apoptosis was quantified by flow cytometry (BD Biosciences, Inc.) analysis.

Cell viability assay. Cell viability was detected by the Cell Counting Kit-8 (CCK-8; Dojindo Molecular Technologies, Inc.). Briefly, the parental cells or drug-resistant cells $\left(2 \times 10^{5}\right.$ cells $\left./ \mathrm{ml}\right)$ were plated into $96-$ well plates. After adherence, the cells were treated with ADR/BOR/DEX at the increasing concentrations of $0,1,5,10,20,40$ and $80 \mathrm{nM}$ for $48 \mathrm{~h}$ at $37^{\circ} \mathrm{C}$. At $2 \mathrm{~h}$ before test, $10 \mu \mathrm{lCCK}-8$ reagent and $150 \mu \mathrm{l}$ DMSO were added into the medium. For the drug sensitivity analysis, the cells were transfected with HMGB1 shRNA and 

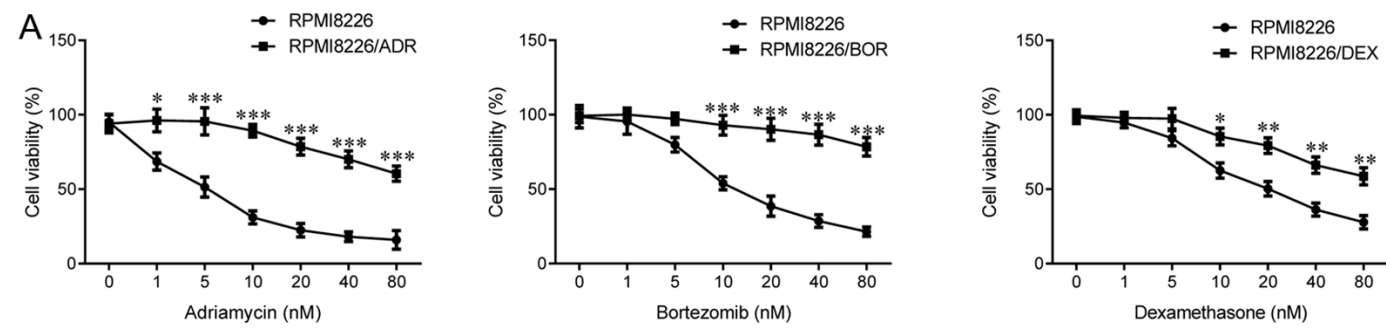

$\mathrm{B}$
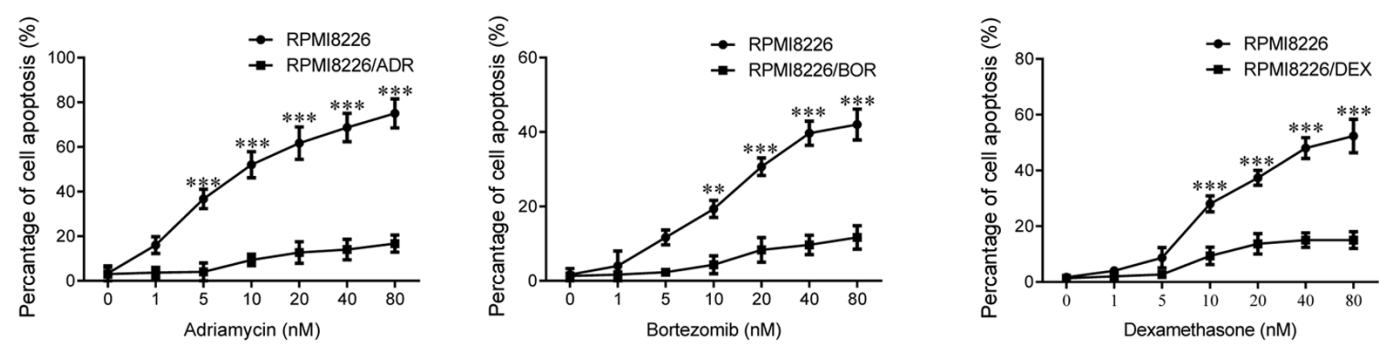

C

Dip $G_{1} \quad$ Dip $S \quad$ Dip $G_{2}$
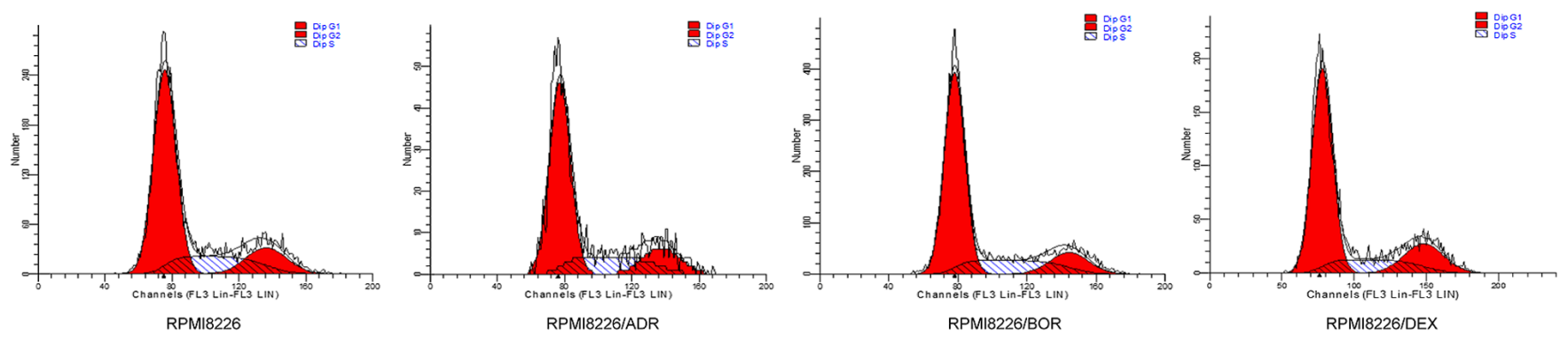

Figure 1. Establishment of multiple myeloma cell lines resistant to adriamycin, bortezomib and dexamethasone. Chemotherapy-sensitive cells, RPMI8226, and three chemotherapy-resistant cells, RPMI8226/ADR, RPMI8226/BOR and RPMI8226/DEX cells, were treated with increasing concentration of adriamycin, bortezomib and dexamethasone for $48 \mathrm{~h}$. (A) The cell viability was determined using the Cell Counting Kit-8 assay. (B) The extent of cell apoptosis was analyzed by measuring Annexin V-positive cells by flow cytometry. (C) The cell cycle was measured by flow cytometric analysis. Data are presented as mean $\pm \mathrm{SD}$ from three independent experiments. ${ }^{*} \mathrm{P}<0.05,{ }^{* *} \mathrm{P}<0.01,{ }^{* * * *} \mathrm{P}<0.001$, vs RPMI8226. ADR, adriamycin; BOR, bortezomib; DEX, dexamethasone.

control shRNA. After $48 \mathrm{~h}$, the cells were treated with ADR/ BOR/DEX at the increasing concentrations of $0,20,40,80,160$, 320 and $640 \mathrm{nM}$ for $48 \mathrm{~h}$ at $37^{\circ} \mathrm{C}$. Absorbance was measured at $490 \mathrm{~nm}$ using a microplate reader (Bio-Rad Laboratories, Inc.; Model 680), and the following formula was used to calculate cell viability: Cell viability $(\%)=\mathrm{OD}$ value of test sample/ OD value of control sample $x 100 \%$. The inhibition rates of chemotherapy drugs on RPMI8226/ADR, RPMI8226/BOR, RPMI8226/DEX cells were performed by CCK-8 assay and $\mathrm{IC}_{50}$ values were calculated using the GraphPad 5.0 software (GraphPad Software, Inc.).

Reverse transcription-quantitative polymerase chain reaction $(R T-q P C R)$. Total RNA was extracted from different cell samples using TRIzol reagent (Invitrogen; Thermo Fisher Scientific, Inc.) according to the manufacturer's instructions and was quantified with a nanophotometer. The obtained RNA samples were reverse-transcribed into single stranded DNA with MLV-reverse transcriptase at $42^{\circ} \mathrm{C}$ for $1 \mathrm{~h}$ (Invitrogen; Thermo Fisher Scientific, Inc.). The Applied Biosystems 7500 Real-time PCR System was used for real-time PCR amplifications using the SYBR Green PCR Master Mix (Applied Biosystems; Thermo Fisher Scientific, Inc.). The PCR thermal cycle conditions were $95^{\circ} \mathrm{C}$ for $1 \mathrm{~min}, 55^{\circ} \mathrm{C}$ for $1 \mathrm{~min}, 72^{\circ} \mathrm{C}$ for $1 \mathrm{~min}$ for $25-30$ cycles, and $72^{\circ} \mathrm{C}$ for $10 \mathrm{~min}$. The relative expression levels of genes was calculated using the $2^{-\Delta \Delta \mathrm{Cq}}$ method (27). The primers used were as follows: HMGB1 forward, 5'-ATATGGCAAAAGCGGACAAG-3'; HMGB1 reverse, 5'-GCAACATCACCAATGGACAG-3'; NF-кB forward, 5'-AGGGCCAGAGACGGATATGT-3'; NF-кB reverse, 5-AGATGTTAGTTGGGCGGTGG'-3'; $\beta$-actin forward, 5'-ACTCGTCATACTCCTGCT-3' and $\beta$-actin reverse, 5'-GAAACTACCTTCAACTCC-3'.

Western blot analysis. Cell lysates were prepared using RIPA buffer [Thermo Fisher Scientific, Inc.; $20 \mathrm{mmol} / 1$ Tris-HCl, $\mathrm{pH} 7.5 ; 150 \mathrm{mmol} / 1 \mathrm{NaCl} ; 1 \mathrm{mmol} / \mathrm{l} \mathrm{Na} 2 \mathrm{EDTA} ; 1 \mathrm{mmol} / \mathrm{l}$ EGTA; $1 \%$ Triton; $2.5 \mathrm{mmol} / 1$ sodium pyrophosphate; $1 \mathrm{mmol} / 1 \mathrm{~b}$-glycerophosphate; $1 \mathrm{mmol} / 1 \mathrm{Na} 3 \mathrm{VO} 4 ; 1 \mathrm{mg} / \mathrm{ml}$ leupeptin; $1 \mathrm{mmol} / 1$ phenylmethylsulfonylfluoride (PMSF); and $1 \mathrm{mmol} / \mathrm{PMSF}$ ] in the presence of a protease inhibitor and PhosStop (Roche Diagnostics). Cytoplasmic and nuclear proteins were respectively isolated using a ProteoJET Cytoplasmic and Nuclear Protein Extraction kit (Thermo Fisher Scientific, Inc.). Protein from cultured medium was extracted by evaporating the medium. Concentration of the extracted protein was detected by a bicinchoninic acid kit (Beyotime Institute of Biotechnology). Then, proteins 

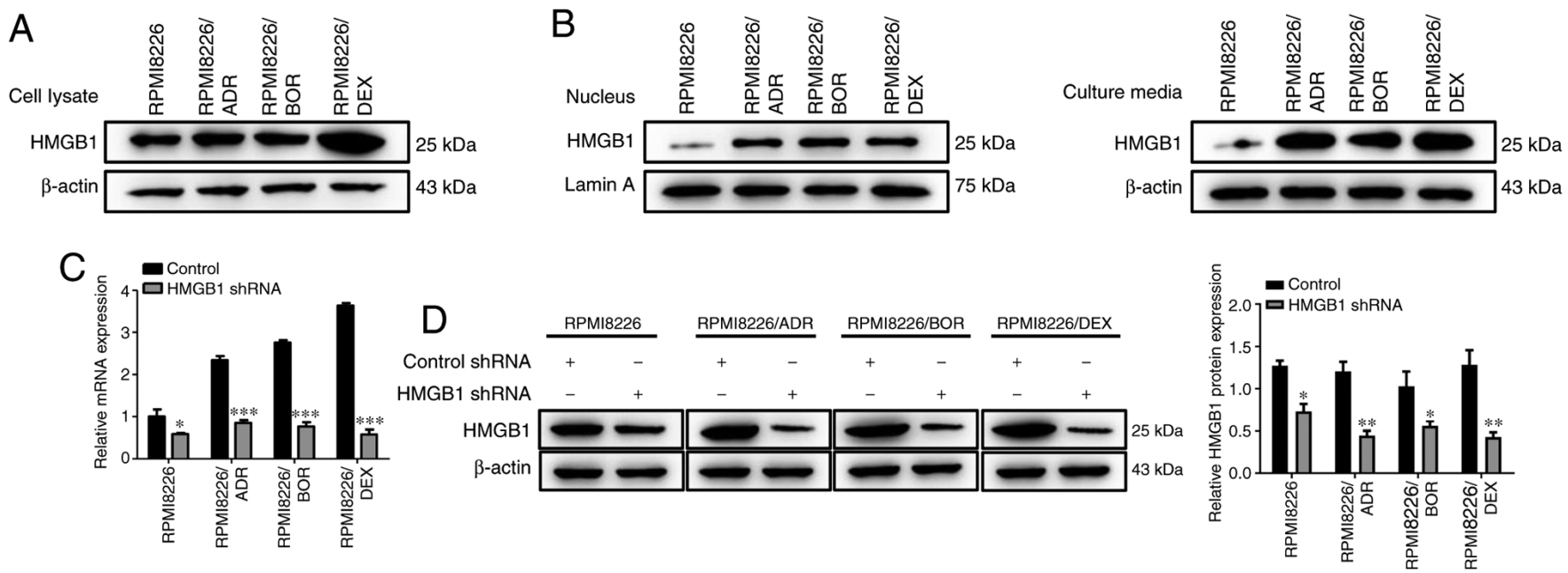

Figure 2. Expression level of HMGB1 is increased in chemotherapy-resistant multiple myeloma cell lines. (A) The HMGB1 protein expression level in the cell lysate of chemotherapy-sensitive cells, RPMI8226, and three chemotherapy-resistant cells, RPMI8226/ADR, RPMI8226/BOR and RPMI8226/DEX cells, were detected by western blotting. $\beta$-actin was used as a loading control. (B) The HMGB1 protein expression level in the nucleus and the culture media of chemotherapy-sensitive cells, RPMI8226, and three chemotherapy-resistant cells, RPMI8226/ADR, RPMI8226/BOR, and RPMI8226/DEX cells was detected by western blotting. (C) The endogenous HMGB1 expression was interfered with shRNA specific to HMGB1. RPMI8226, RPMI8226/ADR, RPMI8226/BOR and RPMI8226/DEX cells were plated into 48-well plate. The cells were cultured overnight and transfected with HMGB1 shRNA and control shRNA for $48 \mathrm{~h}$. Then, the lysates were prepared for detecting HMGB1 expression by reverse transcription-quantitative PCR. (D) The HMGB1 protein expression was detected by western blotting and quantified. $\beta$-actin was used as a loading control. Data are presented as mean $\pm \mathrm{SD}$ of three independent experiments. $\mathrm{P}<0.05,{ }^{* *} \mathrm{P}<0.01$, ${ }^{* * * *} \mathrm{P}<0.001$, vs. RPMI8226. HMGB1, high-mobility group box 1; ADR, adriamycin; BOR, bortezomib; DEX, dexamethasone; shRNA, short hairpin RNA

(20-40 $\mu \mathrm{g})$ were separated on $8-12 \%$ pre-made protein electrophoresis gels and transferred to polyvinylidene difluoride membranes (Merck KGaA). The membranes were blocked with $5 \%$ nonfat milk for $1-2 \mathrm{~h}$ at room temperature. Then, the membranes were incubated with primary antibodies (all purchased from Abcam), including anti-HMGB1 (cat. no. ab18256; 1:1,000), anti-Cylin D1 (cat. no. ab226977; 1:5,000), anti-PCNA (cat. no. ab18197; 1:500), anti-cleaved PARP (cat. no. ab32064; 1:1,000), anti-cleaved caspase-3 (cat. no. ab2302; 1:1,000), anti-TLR4 (cat. no. ab13556; 1:500), anti-p-IKK $\alpha / \beta$ (cat. no. ab194528; 1:500), anti-IKK $\alpha / \beta$ (cat. no. ab178870; 1:1,000), anti-p-IKB $\alpha$ (cat. no. ab133462; 1:1,000), anti-IKB $\alpha$ (cat. no. ab32518; 1:1,000), anti-p-p65 (cat. no. ab86299; 1:20), anti-p65 (cat. no. ab32536; 1:1,000), or anti- $\beta$-actin primary antibodies (cat. no. ab8227; $1: 1,000$ ) overnight at $4^{\circ} \mathrm{C}$. After washes with TBST, the membranes were incubated with secondary antibodies, goat anti-mouse HRP secondary-antibody (cat. no. ab6808; 1:1,000; Abcam) at room temperature for $1 \mathrm{~h}$. The bands were detected using an exposure meter (Bio-Rad Laboratories, Inc.) with an enhanced chemiluminescence detection kit for HRP (Sangon Biotech Co., Ltd.). ImageJ densitometry software (version 1.6, National Institutes of Health) was used to quantitatively analyze western blotting results. $\beta$-actin (cat. no. ab8226; 1:1,000; Abcam) and Lamin A protein (cat. no. MA3-1000; Invitrogen; Thermo Fisher Scientific, Inc.; 1:200) were used as a loading control for cytoplasmic and nuclear proteins.

Statistical analysis. All data are presented as the mean \pm standard deviation (SD) of three replicates per test in a single experiment. A two-tailed Student's t-test was used to determine significant differences between two groups. Tukey's multiple comparison tests was used to analyze the significance of the difference between means. $\mathrm{P}<0.05$ was considered to indicate a statistically significant difference. GraphPad Prism V5.0 was used to perform all statistical analyses.

\section{Results}

Establishment of MMCLs resistant to adriamycin, bortezomib and dexamethasone. To verify the successful establishment of three chemotherapy-resistant RPMI8266 cell lines, CCK-8 assay was conducted to determine the cell viability of parental RPMI8266 and drug-treated RPMI8266 after incubation with the increasing doses $(0,1,5,10,20,40$ and $80 \mathrm{nM})$ of ADR, BOR, and DEX. As shown in Fig. 1A, the cells viability of chemotherapy-resistant RPMI8266 cells was gradually significantly higher compared with that of the parental cells as the drug concentration increased (PRMI8226/ADR, $\mathrm{P}<0.5$ and $\mathrm{P}<0.001$; PRMI8226/BOR, $\mathrm{P}<0.001$; PRMI8226/DEX, $\mathrm{P}<0.05$ and $\mathrm{P}<0.01$ ), which was most notable in RPMI8266/ ADR and RPMI8266/BOR; thus, the drug sensitivity of the three chemotherapy-resistant cells is decreased. Meanwhile, it was found that the drug-resistant cells exhibited a significantly decreased cell apoptosis compared with the parental RPMI8266, in the case of drug treatment ( $\mathrm{P}<0.001$; Fig. 1B). Besides, there was no significant difference in terms of cell cycle between the three chemotherapy-resistant cell lines and the parental RPMI8266 based on the results of flow cytometry analysis (Fig. 1C). In summary, the aforementioned results indicated the successful establishment of ADR-resistant, BOR-resistant and DEX-resistant MMCLs.

Release of HMGB1 is increased from chemotherapy-resistant MMCLs. Next, the expression levels of HMGB1 was detected in the drug-sensitive and drug-resistant MM cells. As shown in Fig. 2A, the protein expression of HMGB1 in the lysate of all three chemotherapy-resistant MMCLs was higher compared 

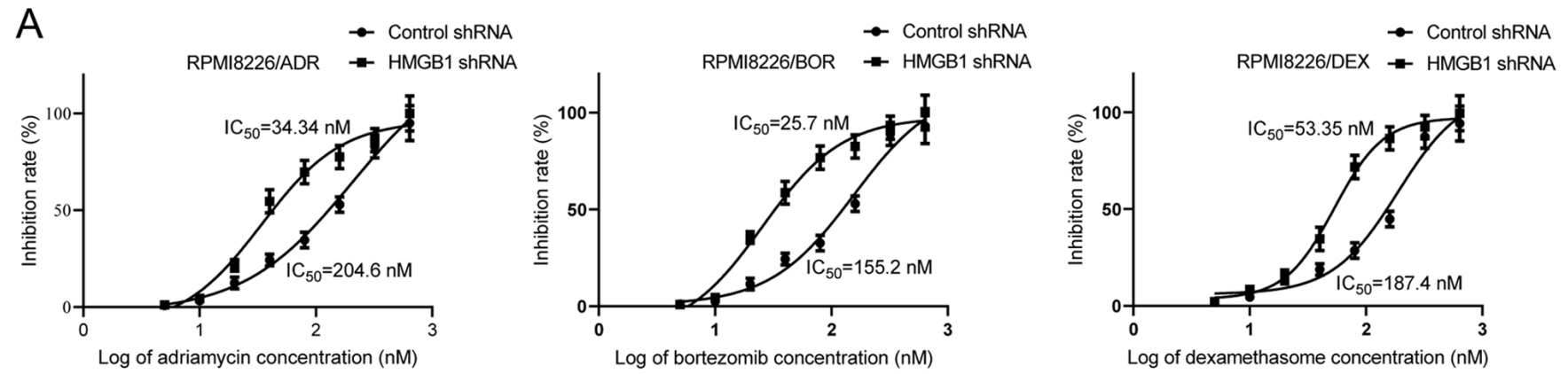

B

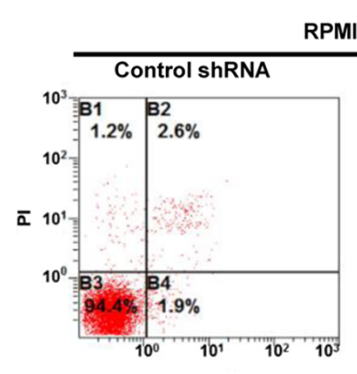

RPMI8226
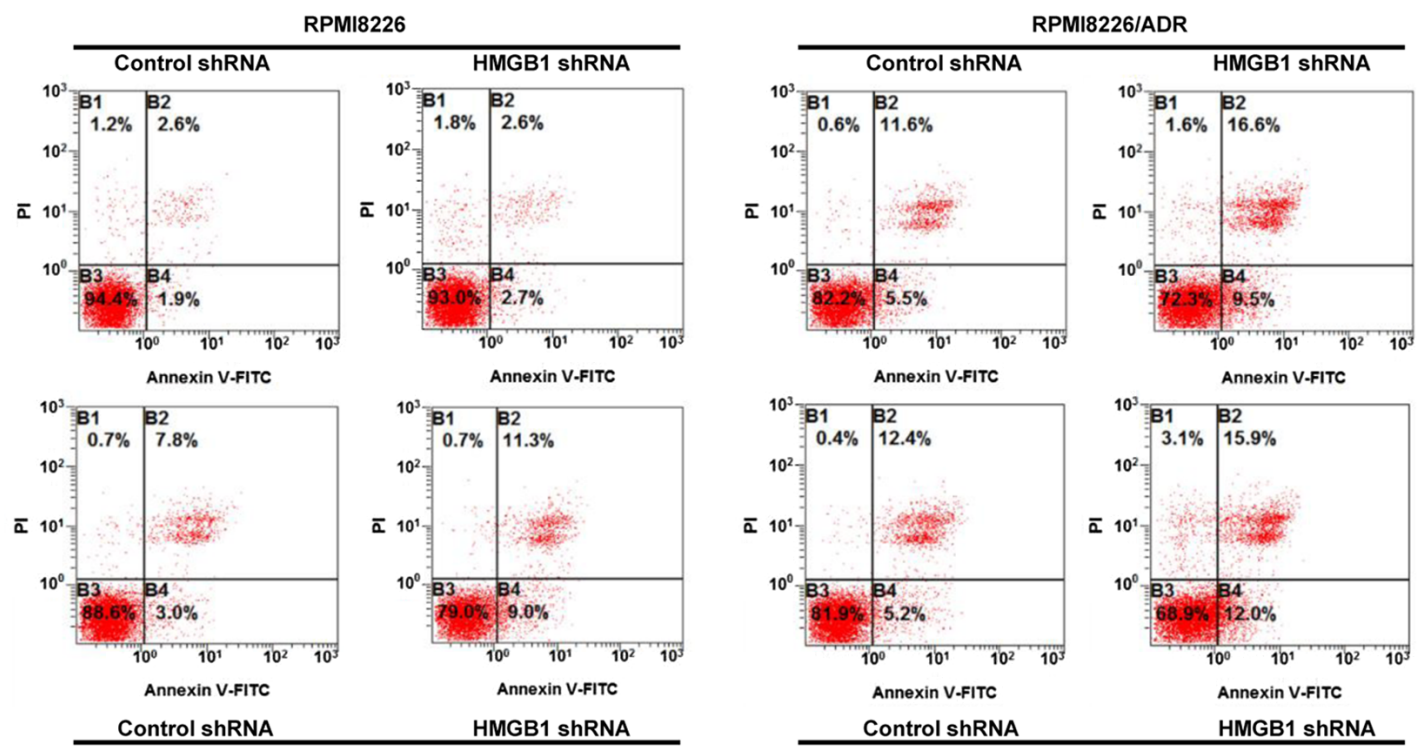

RPMI8226/BOR

RPMI8226/DEX

C

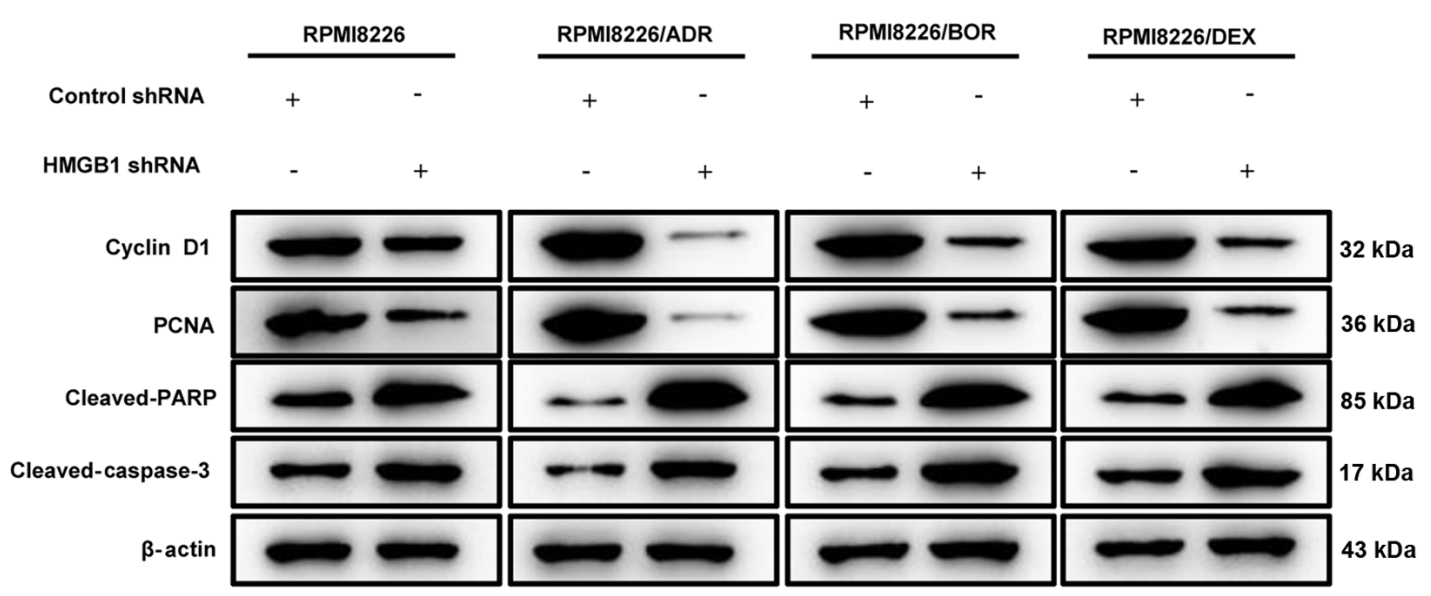

Figure 3. Interference with endogenous HMGB1 increases drug sensitivity in chemotherapy-resistant multiple myeloma cells. Chemotherapy-sensitive cells, RPMI8226, and three chemotherapy-resistant cells, RPMI8226/ADR, RPMI8226/BOR and RPMI8226/DEX cells, were transfected with HMGB1 shRNA and control shRNA. (A) RPMI8226/ADR, RPMI8226/BOR and RPMI8226/DEX were treated with increasing concentrations of ADR, BOR, and DEX, respectively, for $48 \mathrm{~h}$. The inhibition rate was determined by the Cell Cycle Kit-8 assay. The $\mathrm{IC}_{50}$ value was calculated. (B) RPMI8226, RPMI8226/ADR, RPMI8226/ BOR and RPMI8226/DEX cells were respectively treated with a specific concentration of ADR (160 nM), BOR (200 nM) and DEX (180 nM) for 48 h. Cell apoptosis was analyzed by measuring Annexin V-positive cells by flow cytometry. (C) RPMI8226, RPMI8226/ADR, RPMI8226/BOR and RPMI8226/DEX cells were respectively treated with a specific concentration of ADR $(200 \mathrm{nM})$, BOR $(160 \mathrm{nM})$, and DEX $(180 \mathrm{nM})$ for 48 h. The protein expression levels of cyclin D1, PCNA, cleaved-PARP, cleaved-caspase- 3 were detected by western blotting. $\beta$-actin was used as a loading control. Data are presented as mean \pm SD from three independent experiments. HMGB1, high-mobility group box 1; ADR, adriamycin; BOR, bortezomib; DEX, dexamethasone; shRNA, short hairpin RNA.

with that of the chemotherapy-sensitive MM cells. In addition, it was found that the amount of HMGB1 in the drug-resistant
RPMI8226 cells were much higher compared with that in PRMI8226 cells, in both the cell nucleus and culture media; 
moreover, the increase in HMGB1 in the culture media was higher compared with that in the nucleus (Fig. 2B). These findings revealed that chemotherapy-resistant MM cells released higher level of HMGB1 compared with the parental cells, and the increased release of HMGB1 is likely to cause the chemotherapy drug resistance of MM cells; to further verify this, a target-specific shRNA was designed to silence HMGB1 expression. Both RT-qPCR and western blotting results revealed that $\mathrm{HMGB1}$ shRNA transfection contributed to a notable decrease in HMGB1 expression $(\mathrm{P}<0.05, \mathrm{P}<0.01$, $\mathrm{P}<0.001$; Fig. 2C and D), suggesting the successful knockdown of HMGB1 in MM cells.

Interference with endogenous HMGBI increases drug sensitivity in chemotherapy-resistant MM cells. Next, drug sensitivity analysis was performed by treating the three HMGB1 shRNA-transfected chemotherapy-resistant MM cells with increasing concentration of ADR, BOR and DEX. Based on the result of the CCK-8 assay, the $\mathrm{IC}_{50}$ values of HMGB1 shRNA-transfected PRMI8226/ADR, PRMI8226/ BOR and PRMI8226/DEX cells were calculated as 34.34, 25.7 and $53.35 \mathrm{nM}$, respectively, which were significantly lower compared with that of control shRNA-transfected chemotherapy-resistant cells. Thus, interference with endogenous HMGB1 in chemotherapy-resistant MM cells enhances drug sensitivity and decreases resistance (Fig. 3A). Cell apoptosis of RPMI8226, RPMI8226/ADR, RPMI8226/BOR and RPMI8226/DEX cells treated with a specific concentration of ADR (160 nM), BOR (200 nM) and DEX (180 nM) was analyzed by flow cytometry, which found that silencing HMGB1 in drug-resistant MM cells rendered them significantly more sensitive to ADR-, BOR- and DEX-induced cell apoptosis (Fig. 3B). The aforementioned finding was also supported by the decrease in cyclin D1 and PCNA protein expression, and the activation of the pro-apoptotic protein cleaved PARP and cleaved caspase-3 (Fig. 3C). Overall, the data suggest that interference of HMGB1 in chemotherapyresistant $\mathrm{MM}$ cells significantly improved their drug sensitivity.

Interference with endogenous $H M G B 1$ inhibits $N F-\kappa B$ signaling activity in chemotherapy-resistant MM cells. The important role of $\mathrm{NF}-\kappa \mathrm{B}$ signaling in regulating drug sensitivity has been identified in various tumors. Unsurprisingly, a significant increase in TLR4 and NF- $\kappa \mathrm{B}$ mRNA expression was found in all chemotherapy-resistant MM cells $(\mathrm{P}<0.05$, $\mathrm{P}<0.01$; Fig. 4A). Given the high expression of TLR4 and HMGB1 in the MM cells with acquired drug resistance, it was speculated that the enhanced drug sensitivity induced by silencing HMGB1 may be associated with NF- $\mathrm{KB}$ signaling pathway. To further verify this, the effect of HMGB1 on NF-kB activity was next examined. Western blotting showed that HMGB1 shRNA significantly attenuated the TLR4 level, the phosphorylation of IKK $\alpha / \beta$, I $\kappa \mathrm{B} \alpha$, and p 65 in the cell lysate of the three drug-resistant cells compared with the control shRNA cells (Fig. 4B). Moreover, interference with HMGB1 also drastically attenuated the level of phosphorylated p65 in the nucleus ( $\mathrm{P}<0.05 ; \mathrm{P}<0.001$; Fig. 4C). These data revealed an apparent suppression of shRNA HMGB1 on NF- $\kappa \mathrm{B}$ signaling activity, demonstrating that the effect of interference with endogenous HMGB1 on drug sensitivity is most likely because of NF- $\kappa \mathrm{B}$ signaling activity inhibition.

Activation of $N F-\kappa B$ signaling reverses $H M G B 1$ silencinginduced enhancement of chemotherapy sensitivity in $M M$ cells. To further identify the regulatory function of $\mathrm{NF}-\kappa \mathrm{B}$ signaling in the process of HMGB1 silencing-mediated drug sensitivity of MM cells, HY-N6826 (an agonist of NF- $\kappa \mathrm{B}$ signaling pathway) was used to treat the MM cells transfected with HMGB1 shRNA. The results showed that the IKK $\alpha / \beta$, $\mathrm{I} \kappa \mathrm{B} \alpha$ and $\mathrm{p} 65$ in the cell lysate were more phosphorylated in HY-N6826-treated cells in contrast to the cells transfected with HMGB1 shRNA alone (Fig. 5A). Meanwhile, the nuclear translocation of p65 was also significantly increased, as confirmed by the elevation of the nuclear level of phosphorylated p65 (Fig. 5B). These data suggested the successful activation of NF- $\kappa$ B by HY-N6826. Fig. 5C displayed that the treatment of $\mathrm{NF}-\kappa \mathrm{B}$ agonist significantly reversed the inhibition of HMGB1 knockdown on $\mathrm{MM}$ cell viability $(\mathrm{P}<0.05, \mathrm{P}<0.01)$. Moreover, HMGB1 silencing-induced cell apoptosis was also dramatically decreased when $\mathrm{NF}-\kappa \mathrm{B}$ signaling was activated (Fig. 5D; $\mathrm{P}<0.05)$. Similar outcome was also confirmed by the protein expression of cyclin D1, PCNA, cleaved-PARP and cleavedcaspase 3 (Fig. 5E). The aforementioned data manifest that the activation of $\mathrm{NF}-\kappa \mathrm{B}$ signaling could effectively reverse the impact of HMGB1 silencing on the drug sensitivity of MM cells.

\section{Discussion}

As a malignant hematological tumor, $\mathrm{MM}$ is characterized by the abnormal proliferation of plasma cells in the bone marrow, which has been seriously threatened human health and survival (28). Currently, diverse clinical therapies including chemotherapy, targeted therapy and immunotherapy have been used for the treatment of MM, which are all in the face of a challenging problem of drug resistance that has become a major obstacle in successfully treating MM. Although the common anticancer chemotherapy drugs, adriamycin, bortezomib and dexamethasone have exhibited a satisfactory therapeutic outcome in the treatment of MM, the widespread emergence of acquired resistance to chemotherapy greatly limits the use of these drugs (29). Therefore, it is urgent to define the exact mechanism of chemotherapy resistance that is clinically relevant in MM, which may be helpful to predict and overcome drug resistance, thereby improving chemotherapy and ultimately the outcome of patients with cancer.

In order to explore the exact mechanism of drug resistance in MM, three drug-resistant MMCLs were first constructed. Based on the result by which the increased cell viability and the decreased cell apoptosis of drug-resistant MM cells compared with the parental cells, as well as the similar cell cycle between the three chemotherapy-resistant cell lines and the parental cells, the successful establishment of ADR-resistant, BOR-resistant, and DEX-resistant MMCLs was confirmed.

HMGB1 has been found to be overexpressed in a variety of hematological malignancies, such as leukemia and lymphoma (30), and its high expression has been shown to be a strong predictor of poor survival in diverse malignancies, including colorectal cancer, gastric cancer, nasopharyngeal 

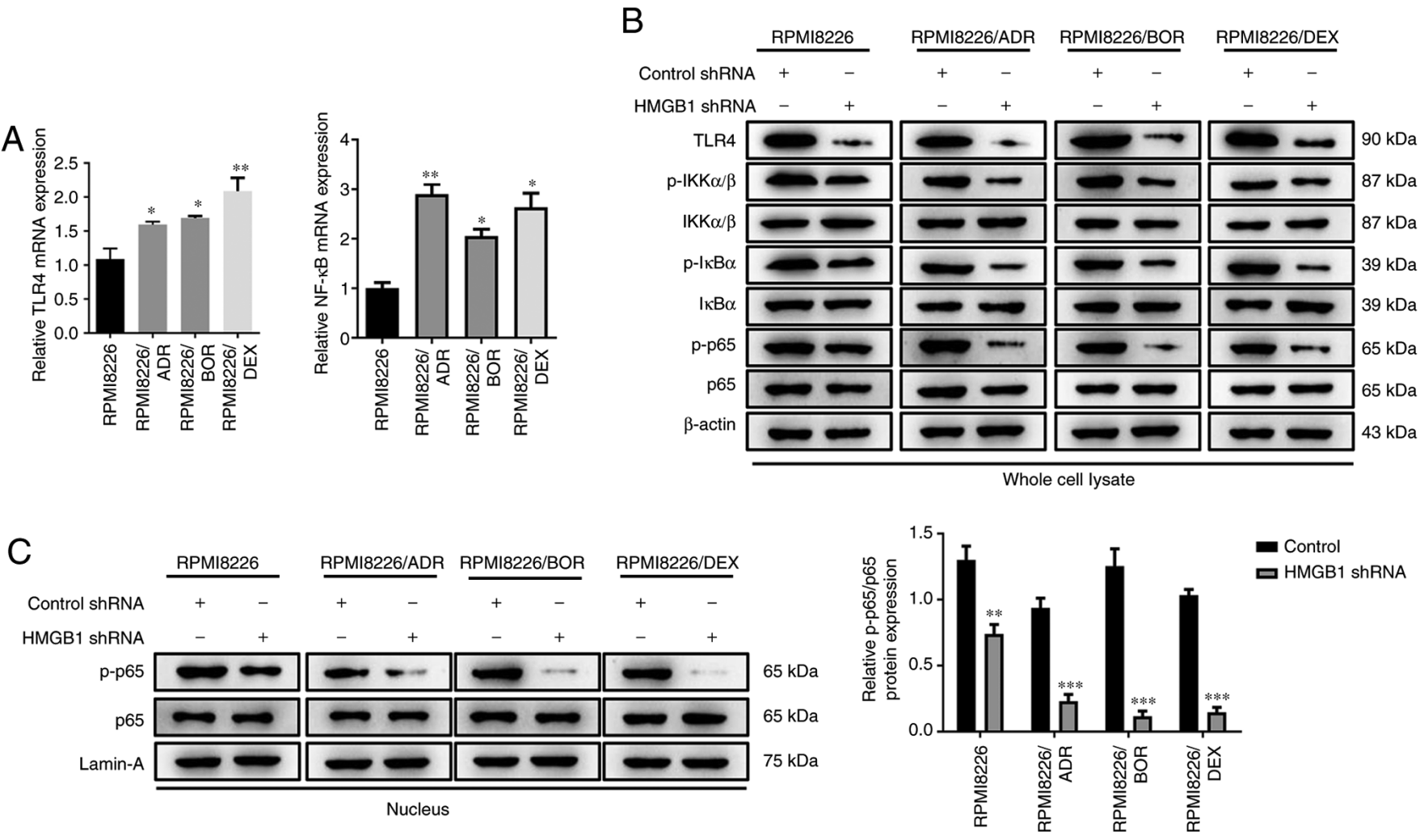

Figure 4. Interference with endogenous HMGB1 inhibits NF- $\kappa$ B signaling activity in chemotherapy-resistant multiple myeloma cells. Chemotherapy-sensitive cells, RPMI8226, and three chemotherapy-resistant cells, RPMI8226/ADR, RPMI8226/BOR and RPMI8226/DEX cells, were transfected with HMGB1 shRNA or control shRNA. (A) The relative mRNA expression of TLR4 and NF- $\kappa$ B mRNA were detected by reverse transcription-quantitative PCR. (B) The

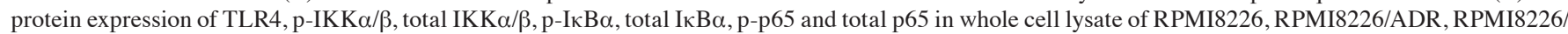
BOR and RPMI8226/DEX cells were detected by western blotting. $\beta$-actin was used as a loading control. (C) The protein expression of p-p65 and total p-65 in the nucleus of RPMI8226, RPMI8226/ADR, RPMI8226/BOR and RPMI8226/DEX cells were detected by western blotting and quantified. Lamin-A was used as a loading control. Data are presented as mean $\pm \mathrm{SD}$ from three independent experiments. ${ }^{*} \mathrm{P}<0.05,{ }^{* *} \mathrm{P}<0.01,{ }^{* * *} \mathrm{P}<0.001 \mathrm{vs}$. RPMI8226. HMGB1, highmobility group box 1; ADR, adriamycin; BOR, bortezomib; DEX, dexamethasone; p-, phosphorylated; shRNA, short hairpin RNA; TLR4, Toll-like receptor $4 ; \mathrm{IKK} \alpha / \beta$, I-к-B kinase $\alpha / \beta$.

carcinoma and squamous-cell carcinoma of the head and neck (31). In MM, a study based on GEP analysis revealed a higher expression level of HMGB1 in the bone marrow plasma cells of patients with MM compared with that in healthy donors, and the high level of HMGB1 also indicated a poor survival in these patients; meanwhile, a significant upregulation of HMGB1 has been observed in bortezomib resistant-MM cells (12). Besides, silencing HMGB1 in MM cells could enhance the inhibitory effect of chemotherapy with dexamethasone via regulating the mTOR pathway (11). In the present study, all three drug-resistant MMCLs expressed an increased expression level of HMGB1 compared with the parental cells; this observation was confirmed by the prior finding of the higher HMGB1 expression in bortezomib-resistant MM cells (11). These findings confirmed a close association between HMGB1 and drug resistance in MM. Actually, several studies had been carried out to explore the association between HMGB1 expression and chemotherapy resistance in various tumor cells. For example, a significantly enhanced expression of HMGB1 was found in cisplatin- and methotrexate-treated human osteosarcoma cells (32); PTX has been shown to induce HMGB1 release from human pancreatic cancer cells and colon cancer cells (33); Liu et al (9) found that chemotherapy treatment significantly improved the level of HMGB1 in the supernatants of leukemia cell cultures (9); the study of Zheng et al (7) revealed that
HMGB1 levels were much higher in the cisplatin-resistant cell line compared with the cisplatin-sensitive cell line, and the levels of HMGB1 level in cytoplasm gradually elevated with the increasing concentrations of cisplatin.

To further explore the regulatory role of HMGB1 in drug resistance in MM cells, an shRNA was designed to interfere with the expression of HMGB1. The results of CCK-8 and flow cytometry assays manifested that HMGB1 knockdown significantly decreased the $\mathrm{IC}_{50}$ value and promoted cell apoptosis of drug-resistant MM cells; this finding suggests that HMGB1 might be a driver to promote MM cells to develop chemotherapy drug resistance. Based on a previous study (34), it is postulated that the mechanisms of drug-resistance causing an increase in HMGB1 in myeloma cells may be due to that the chemotherapeutic drugs-induced autophagy in MM cells, and the translocation of HMGB1 from the nucleus to the cytoplasm is a key molecular event in this process. Previous studies provided empirical evidence that extracellular HMGB1 is associated with both sensitivity and resistance to anticancer therapy. The present finding was consistent with the findings by Zheng et al (7) that knockdown of HMGB1 enhanced the chemosensitivity of human lung cancer cells to chemotherapeutic drugs, including 5-FU, DDP and OXA. Similarly, an associated study showed that the blockage of HMGB1 release could effectively inhibit cell viability and strengthen the 


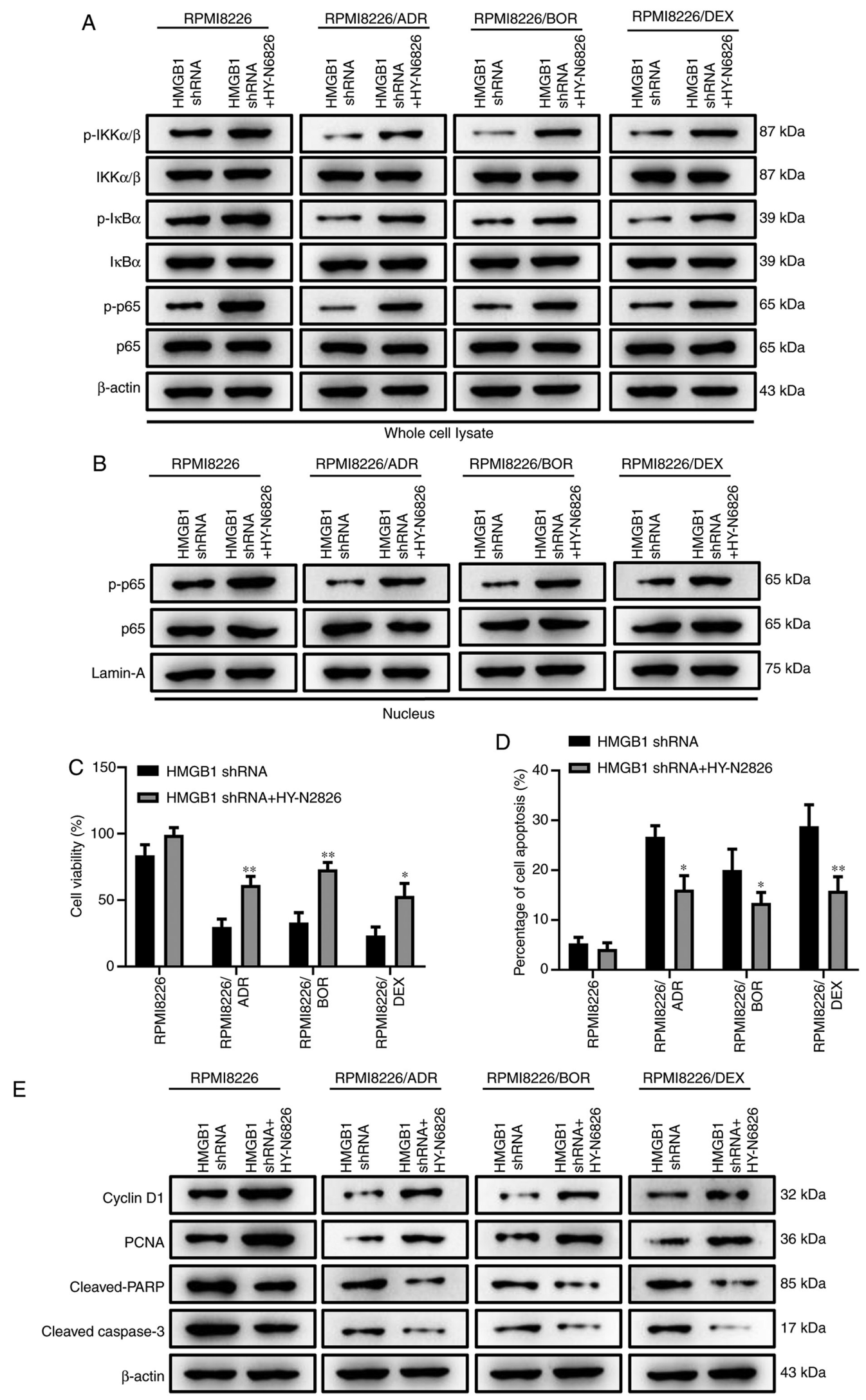

Figure 5. Activation of NF- $\mathrm{KB}$ signaling reverses HMGB1 silencing-induced enhancement of chemotherapy sensitivity in multiple myeloma cells. The MM cells transfected with HMGB1 shRNA were treated with an agonist of nuclear factor- $\mathrm{\kappa B}$ signaling pathway, HY-N6826 (asatone). (A) The protein expression

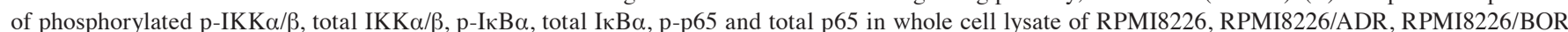
and RPMI8226/DEX cells were detected by western blotting. $\beta$-actin was used as a loading control. (B) The protein expression of p-p65 and total p-65 in the nucleus of RPMI8226, RPMI8226/ADR, RPMI8226/BOR and RPMI8226/DEX cells were detected by western blotting. Lamin-A was used as a loading control. (C) The cell viability was determined using the Cell Counting Kit-8 assay. (D) The quantification of Annexin-positive cells based on the result of flow cytometry assay. (E) The protein expression of cyclin D1, PCNA, cleaved-PARP, cleaved-caspase-3 was detected by western blotting. $\beta$-actin was used as a loading control. Data are presented as mean $\pm \mathrm{SD}$ from three independent experiments. ${ }^{*} \mathrm{P}<0.05,{ }^{* *} \mathrm{P}<0.01 \mathrm{vs}$. RPMI8226.HMGB1, high-mobility group box 1 ; ADR, adriamycin; BOR, bortezomib; DEX, dexamethasone; p-, phosphorylated; shRNA, short hairpin RNA; IKK $\alpha / \beta$, I-k-B kinase $\alpha / \beta$. 
sensitivity of DU145R cells to PTX, indicating a promotion of PTX resistance induced by the released HMGB1 in prostate cancer cells (35). Meanwhile, HMGB1 overexpression can attenuate the leukemia cell sensitivity to adriamycin, vincristine and cytarabine, while HMGB1 knockdown enhanced this effect (36). Of note, if the drug sensitivity analysis was also performed in HMGB1-transfected cells, the confidence of the present results may be higher; this can be considered as a minor limitation of the present study, and would be served as the research focus in the future study.

HMGB1 a DNA binding protein located in nucleus, has been identified to participate in the regulation of drug resistance and sensibility in various tumors via the mediation of various signaling pathways. For example, HMGB1 can promote the formation and fusion of the autophagosome with the lysosome by activating the PI3K-MEK-ERK pathway, thereby enhancing the resistance of leukemia cells to anticancer therapies (9). Moreover, overexpression HMGB1 induces drug resistance of the leukemia cells by inducing autophagy via the PI3K/Akt/mTORC1 pathway, while knockdown HMGB1 inhibiting leukemia cell autophagy and increasing drug sensitivity through increasing the phosphorylation of Akt and p70S6k (9,36). As a novel proinflammatory cytokine, HMGB1 has been demonstrated to interact with binding to Toll-like receptor 4 (TLR4) to activate NF- $\kappa \mathrm{B}$ (37). In the present study, the interference with endogenous HMGB1 was found to remarkably decrease TLR4 expression and $\mathrm{NF}-\kappa \mathrm{B}$ signaling activity, which is consistent with the previous study (38). Activated $\mathrm{NF}-\kappa \mathrm{B}$ has been considered as an important role of cisplatin resistance, which could negatively regulate the cellular sensitivity of carcinoma cell lines to chemotherapy (38). Furthermore, the cisplatin-resistant lung cancer cells displayed an elevated $\mathrm{NF}-\kappa \mathrm{B}$ expression in contrast to their parental cells, supporting a potential regulatory role of $\mathrm{NF}-\kappa \mathrm{B}$ in acquired cisplatin resistance (21). Based on the aforementioned finding, it was hypothesized that there is a potential association between HMGB1-mediated drug resistance and $\mathrm{NF}-\kappa \mathrm{B}$ signaling pathway.

To confirm the hypothesis, further rescue experiments were performed and the results showed that the activation of $\mathrm{NF}-\kappa \mathrm{B}$ signaling reverses HMGB1 silencing-induced enhancement of drug sensitivity in MM cells. Numerous studies have explored the regulatory association between HMGB1 and NF- $\mathrm{BB}$ signaling. van Beijnum et al (39) found that HMGB1 administration can activate $\mathrm{NF}-\kappa \mathrm{B}$ pathway via TLR4, thereby involving the regulation of inflammation and activation of immune cells. The study by Huang et al (40) indicated that HMGB1 silencing significantly inhibited the activation of $\mathrm{NF}-\kappa \mathrm{B}$ by suppressing the nuclear translocation and DNA-binding activity of NF- $\mathrm{NB} / \mathrm{p} 65$. In murine models, the treatment of cells with an AKT-NF-kB inhibitor (genistein) rendered them sensitive to cisplatin-induced apoptosis by increasing NF- $\kappa \mathrm{B}$ activity (41).

In conclusion, based on the establishment of three drugresistant MMCLs, high expression of HMGB1 was observed in the chemotherapy-resistant MM cells, and the interference of endogenous HMGB1 enhanced the drug sensibility and inhibited $\mathrm{NF}-\kappa \mathrm{B}$ signaling activity in chemotherapy-resistant MM cells. The activation of $N F-\kappa B$ signaling restored HMGB1 silencing-induced enhancement of drug sensitivity, manifesting a significant role of $\mathrm{NF}-\kappa \mathrm{B}$ signaling pathway in HMGB1-mediated drug resistance. These results provided a greater understanding of the potential molecular mechanism of HMGB1 in regulating drug resistance of MM cells, which may be helpful for the development of novel therapeutic strategies and drugs to treat MM.

\section{Acknowledgements}

Not applicable.

\section{Funding}

This study was supported by Ningxia Hui Autonomous Region Key Research and Development Project (grant no. 2019BEG03053).

\section{Availability of data and materials}

All data generated or analyzed during this study are included in this published article.

\section{Authors' contributions}

JN, RY, HW and LC initiated the work and designed the experiments. JN performed the majority of the experiments. JN and RY wrote the manuscript. HW and LC provided samples and critical suggestions. All authors read and approved the final manuscript. JN and LC confirm the authenticity of all the raw data.

\section{Ethics approval and consent to participate}

Not applicable.

\section{Patient consent for publication}

Not applicable.

\section{Competing interests}

The authors declare that they have no competing interests.

\section{References}

1. Sparano F, Cavo M, Niscola P, Caravita T and Efficace F: Patientreported outcomes in relapsed/refractory multiple myeloma: A systematic review. Support Care Cancer 26: 2075-2090, 2018.

2. McCullough KB, Hobbs MA, Abeykoon JP and Kapoor P: Common adverse effects of novel therapies for multiple myeloma (MM) and Their management strategies. Curr Hematol Malig Rep 13: 114-124, 2018.

3. Abdi J, Chen G and Chang H: Drug resistance in multiple myeloma: Latest findings and new concepts on molecular mechanisms. Oncotarget 4: 2186-2207, 2013.

4. Raucci A, Di Maggio S, Scavello F, D'Ambrosio A, Bianchi ME and Capogrossi MC: The Janus face of HMGB1 in heart disease: A necessary update. Cell Mol Life Sci 76: 211-229, 2019.

5. Venereau E, De Leo F, Mezzapelle R, Careccia G, Musco G and Bianchi ME: HMGB1 as biomarker and drug target. Pharmacol Res 111: 534-544, 2016.

6. Yang H, Antoine DJ, Andersson U and Tracey KJ: The many faces of HMGB1: Molecular structure-functional activity in inflammation, apoptosis, and chemotaxis. J Leukoc Biol 93: 865-873, 2013 
7. Zheng H, Chen JN, Yu X, Jiang P, Yuan L, Shen HS, Zhao LH, Chen PF and Yang M: HMGB1 enhances drug resistance and promotes in vivo tumor growth of lung cancer cells. DNA Cell Biol 35: 622-627, 2016.

8. Zhang YX, Yuan YQ, Zhang XQ, Huang DL, Wei YY and Yang JG: HMGB1-mediated autophagy confers resistance to gemcitabine in hormone-independent prostate cancer cells. Oncol Lett 14: 6285-6290, 2017.

9. Liu L, Yang M, Kang R, Wang Z, Zhao Y, Yu Y, Xie M, Yin X, Livesey KM, Lotze MT, et al: HMGB1-induced autophagy promotes chemotherapy resistance in leukemia cells. Leukemia 25: 23-31, 2011.

10. Pan B, Chen D, Huang J, Wang R, Feng B, Song H and Chen L: HMGB1-mediated autophagy promotes docetaxel resistance in human lung adenocarcinoma. Mol Cancer 13: 165, 2014.

11. Guo X, He D, Zhang E, Chen J, Chen Q, Li Y, Yang L, Yang Y, Zhao Y, Wang G et al: HMGB1 knockdown increases MM cell vulnerability by regulating autophagy and DNA damage repair. J Exp Clin Cancer Res 37: 205, 2018.

12. Roy M, Liang L, Xiao X, Peng Y, Luo Y, Zhou W, Zhang J, Qiu L, Zhang S, Liu F, et al: Lycorine downregulates HMGB1 to inhibit autophagy and enhances bortezomib activity in multiple myeloma. Theranostics 6: 2209-2224, 2016.

13. Hanahan D and Weinberg RA: Hallmarks of cancer: The next generation. Cell 144: 646-674, 2011

14. Mansouri L, Papakonstantinou N, Ntoufa S, Stamatopoulos K and Rosenquist R: NF- $\mathrm{BB}$ activation in chronic lymphocytic leukemia: A point of convergence of external triggers and intrinsic lesions. Semin Cancer Biol 39: 40-48, 2016.

15. Tew GW, Lorimer EL, Berg TJ, Zhi H, Li R and Williams CL: SmgGDS regulates cell proliferation, migration, and NF-kappaB transcriptional activity in non-small cell lung carcinoma. J Biol Chem 283: 963-976, 2008.

16. Boudesco C, Verhoeyen E, Martin L, Chassagne-Clement C, Salmi L, Mhaidly R, Pangault C, Fest T, Ramla S, Jardin F, et al HSP110 sustains chronic NF- $\mathrm{B}$ signaling in activated B-cell diffuse large B-cell lymphoma through MyD88 stabilization. Blood 132: 510-520, 2018

17. Chua HL, Bhat-Nakshatri P, Clare SE, Morimiya A, Badve S and Nakshatri H: NF-kappaB represses E-cadherin expression and enhances epithelial to mesenchymal transition of mammary epithelial cells: Potential involvement of ZEB-1 and ZEB-2 Oncogene 26: 711-724, 2007.

18. Kina I, Sultuybek GK, Soydas T, Yenmis G, Biceroglu H, Dirican A, Uzan M and Ulutin T: Variations in Toll-like recepto and nuclear factor-kappa B genes and the risk of glioma. Br J Neurosurg 33: 165-170, 2019.

19. Rada M, Nallanthighal S, Cha J, Ryan K, Sage J,Eldred C, Ullo M, Orsulic S and Cheon DJ: Inhibitor of apoptosis proteins (IAPs) mediate collagen type XI alpha 1-driven cisplatin resistance in ovarian cancer. Oncogene 37: 4809-4820, 2018.

20. Seubwai W, Vaeteewoottacharn K, Kraiklang R, Umezawa K Okada S and Wongkham S: Inhibition of NF- $\kappa \mathrm{B}$ activity enhances sensitivity to anticancer drugs in cholangiocarcinoma cells. Oncol Res 23: 21-28, 2016.

21. Barr MP, Gray SG, Hoffmann AC, Hilger RA, Thomale J, O'Flaherty JD, Fennell DA, Richard D, O'Leary JJ and O'Byrne KJ: Generation and characterisation of cisplatinresistant non-small cell lung cancer cell lines displaying a stem-like signature. PLoS One 8: e54193, 2013

22. Broyl A, Hose D, Lokhorst H, de Knegt Y, Peeters J, Jauch A, Bertsch U, Buijs A, Stevens-Kroef M, Beverloo HB, et al: Gene expression profiling for molecular classification of multiple myeloma in newly diagnosed patients. Blood 116: 2543-2553, 2010.

23. Peng H, Peng T, Wen J, Engler DA, Matsunami RK, Su J, Zhang L, Chang CC and Zhou X: Characterization of p38 MAPK isoforms for drug resistance study using systems biology approach. Bioinformatics 30: 1899-1907, 2014.

24. Demchenko YN, Glebov OK, Zingone A, Keats JJ, Bergsagel PL and Kuehl WM: Classical and/or alternative NF-kappaB pathway activation in multiple myeloma. Blood 115: 3541-3552, 2010.
25. Keats JJ, Fonseca R, Chesi M, Schop R, Baker A, Chng WJ, Van Wier S, Tiedemann R, Shi CX, Sebag M, et al: Promiscuous mutations activate the noncanonical NF-kappaB pathway in multiple myeloma. Cancer Cell 12: 131-144, 2007.

26. Zhang J, Shao S, Han D, Xu Y, Jiao D, Wu J, Yang F, Ge Y, Shi S, Li Y, et al: High mobility group box 1 promotes the epithelialto-mesenchymal transition in prostate cancer PC 3 cells via the RAGE/NF-KB signaling pathway. Int J Oncol 53: 659-671, 2018.

27. Livak KJ and Schmittgen TD: Analysis of relative gene expression data using real-time quantitative PCR and the 2(-Delta Delta C(T)) Method. Methods 25: 402-408, 2001

28. Choi YW, Park JS, Han JH, Kim JH, Ahn MS, Lee HW, Kang SY, Choi JH and Jeong SH: Strong immunoexpression of dickkopf-1 is associated with response to bortezomib in multiple myeloma. Leuk Lymphoma 59: 2670-2678, 2018.

29. Zhang Y, Liu H, Chen X, Bai Q, Liang R, Shi B, Liu L, Tian D and Liu M: Modified bortezomib, adriamycin and dexamethasone (PAD) regimen in advanced multiple myeloma. Pathol Oncol Res 20: 987-995, 2014

30. Sohun M and Shen H: The implication and potential applications of high-mobility group box 1 protein in breast cancer. Ann Transl Med 4: 217, 2016.

31. Murakami T, Matsuyama R, Ueda M, Mochizuki Y, Homma Y, Kameda K, Yazawa K, Izumisawa Y, Fukushima T, Kamimukai N, et al: High-Mobility Group Box 1 expression predicts survival of patients after resection of adenocarcinoma of the ampulla of Vater. World J Surg Oncol 17: 140, 2019.

32. Huang J, Ni J, Liu K, Yu Y, Xie M, Kang R, Vernon P, Cao L and Tang D: HMGB1 promotes drug resistance in osteosarcoma. Cancer Res 72: 230-238, 2012.

33. Aldonza MB, Hong JY and Lee SK: Paclitaxel-resistant cancer cell-derived secretomes elicit ABCB1-associated docetaxel cross-resistance and escape from apoptosis through FOXO3adriven glycolytic regulation. Exp Mol Med 49: e286, 2017.

34. Li Y, Xie J, Li X and Fang J: Poly (ADP-ribosylation) of HMGB1 facilitates its acetylation and promotes HMGB1 translocationassociated chemotherapy-induced autophagy in leukaemia cells. Oncol Lett 19: 368-378, 2020

35. Zhou J, Chen X, Gilvary DL, Tejera MM, Eksioglu EA, Wei S and Djeu JY: HMGB1 induction of clusterin creates a chemoresistant niche in human prostate tumor cells. Sci Rep 5: 15085, 2015.

36. Yang L, Yu Y, Kang R, Yang M, Xie M, Wang Z, Tang D, Zhao M, Liu L, Zhang H, et al: Up-regulated autophagy by endogenous high mobility group box-1 promotes chemoresistance in leukemia cells. Leuk Lymphoma 53: 315-322, 2012.

37. Shi Y, Zhang L, Teng J and Miao W: HMGB1 mediates microglia activation via the TLR4/NF- $\kappa$ B pathway in coriaria lactone induced epilepsy. Mol Med Rep 17: 5125-5131, 2018.

38. Almeida LO, Abrahao AC, Rosselli-Murai LK, Giudice FS, Zagni C, Leopoldino AM, Squarize CH and Castilho RM: NFKB mediates cisplatin resistance through histone modifications in head and neck squamous cell carcinoma (HNSCC). FEBS Open Bio 4: 96-104, 2013

39. van Beijnum JR, Buurman WA and Griffioen AW: Convergence and amplification of toll-like receptor (TLR) and receptor for advanced glycation end products (RAGE) signaling pathways via high mobility group B1 (HMGB1). Angiogenesis 11:91-99, 2008.

40. Huang Z, Zhong Z, Zhang L, Wang X, Xu R, Zhu L, Wang Z, $\mathrm{Hu} \mathrm{S}$ and Zhao X: Down-regulation of HMGB1 expression by shRNA constructs inhibits the bioactivity of urothelial carcinoma cell lines via the NF- $\kappa B$ pathway. Sci Rep 5: 12807, 2015.

41. Li Y, Ahmed F, Ali S, Philip PA, Kucuk O and Sarkar FH: Inactivation of nuclear factor kappaB by soy isoflavone genistein contributes to increased apoptosis induced by chemotherapeutic agents in human cancer cells. Cancer Res 65: 6934-6942, 2005.

This work is licensed under a Creative Commons Attribution-NonCommercial-NoDerivatives 4.0 International (CC BY-NC-ND 4.0) License. 